\title{
Cost-based Procedure for Multi-response Parameter Design Problems Using GEP, Taguchi Quality Loss, and PSO: Case Study on Heat Sink Design
}

\author{
Chih-Ming Hsu \\ Department of Business Administration, Minghsin University of Science and Technology, 1 Hsin-Hsing Rd., Hsin-Fong, \\ Hsinchu, 304, Taiwan, R.O.C. \\ Received 2 March 2014
}

Accepted 18 August 2014

\begin{abstract}
Most modern products/processes usually have several quality characteristics that must be optimized simultaneously; this is called a multi-response parameter design problem. To overcome shortcomings in the literature, including insufficient accuracy of second-order polynomials, subjective determination of relative weights and shape coefficients, and non-consideration of manufacturing or material costs, this paper proposes a cost-based procedure for resolving multi-response parameter design problems using gene expression programming (GEP), Taguchi quality loss, and particle swarm optimization (PSO). A case study with the aim of optimizing the design of a heat sink applied to a high-power MR16 LED lamp was used to demonstrate the proposed procedure. The experimental results indicated that the proposed procedure can provide highly robust settings for design parameters that can maximize the thermal performance and minimize the actual material cost of a heat sink. Furthermore, decisionmakers no longer need to subjectively determine the relative weight of each response. Therefore, the proposed approach can be considered to be feasible and effective; it has the potential to be a useful tool for resolving general multi-response parameter design problems in the real world.
\end{abstract}

Keywords: Heat sink; Gene expression programming; Taguchi quality loss; Particle swarm optimization; Multiresponse parameter design

\section{Introduction}

A heat sink is a passive heat exchanger component that cools a device by dissipating heat into the surrounding air. Fig. 1 shows a typical heat sink used in a highpower MR16 LED (light emitting diode) lamp. To simultaneously minimize the material cost and maximize the thermal performance of a heat sink, the material, fin design, surface treatment, etc. must be determined conservatively; this is considered to be a complicated multi-response parameter design problem.

The Taguchi method is a well-known traditional approach for tackling such a problem; however, it has not proved to be fully functional for optimizing multiple responses, especially in the case of correlated responses [1]. Therefore, some approaches have been incorporated with the Taguchi method to determine the discrete settings of control factors based on their original experimental levels [e.g., 2-9].

However, the true optimal parameter settings of control factors may exist throughout the entire experimental range. Consequently, many recent studies have focused on using/integrating techniques from various fields to find the continuous settings of control factors for a multi-response parameter design problem. For example, Kim and Lin [10] applied response surface methodology (RSM) and exponential desirability functions to maximize the overall minimal value of satisfaction with respect to all responses to determine the optimal settings of control factors. Kovach and Cho [11] utilized a combined array design to incorporate noise factors into the robust design model and used response surface functions to describe the functional dependence of the mean and variance of each quality characteristic on the control factors. The system specifications and desired target values were then 
incorporated as constraints and goals, respectively. Finally, a nonlinear goal programming technique with the first goal of minimizing the variance and second goal of attempting to make the mean equal to the desired target value was employed to optimize multiple responses simultaneously. Ramezani et al. [12] used the prediction intervals from the model building stage and applied the concepts of the goal programming approach with normalization based on negative and positive ideal solutions to generate a set of non-dominated and efficient solutions. The non-dominated solutions were then ranked using the technique for order preference by similarity to ideal solution (TOPSIS) to provide some suggested settings for control factors. Sibalija et al. [1] evaluated the overall performance regarding all responses via a synthetic measure generated by using Taguchi's quality losses, principal component analysis (PCA), and grey relational analysis (GRA). The genetic algorithms (GAs) were then utilized to determine the optimal parameter settings of control factors by searching the mathematical model that was described through neural networks (NNs). Bera and Mukherjee [13] proposed an adaptive penalty function-based "maximin" desirability index for multiple-response optimization problems with close engineering tolerances of quality characteristics and determined a near-optimal solution using continuous ant colony optimization, ant colony optimization in real space, and global best particle swarm optimization. Devi et al. [14] proposed a Taguchi-based modified epsilon constraint method to obtain a non-dominated Pareto solution set for a multiobjective design optimization where the objective functions to be optimized were described by linear regression equations derived from the Taguchi experimental design. Goethals and Cho [15] applied higher-order estimation techniques instead of traditional second-order equations to model the mathematical relationship between control factors and responses and incorporated the concepts of robust design to the desirability function to account for process variability and thus determine the optimal settings for the control factors. He et al. [16] considered all values in the confidence interval rather than a single predicted value for each response and applied the worst-case strategy to define the robustness measure for the traditional desirability function and consider the uncertainty associated with the fitted response surface model. A hybrid genetic algorithm coupled with pattern search was then used to find the robust optimal solution. Mukherjee et al. [17] used RSM-based second-order equations to model the considered responses and applied six popular population-based optimization algorithms - genetic algorithms (GAs), particle swarm optimization (PSO), sheep flock (SF) algorithm, ant colony optimization (ACO), artificial bee colony (ABC), and biogeography-based optimization (BBO) - to obtain the optimal settings for the input control factors of two wire electrical discharge machining (WEDM) processes. A comparison of the results revealed that BBO outperformed the others. Salmasnia et al. [18] presented a three-phased approach that uses principal component analysis (PCA), adaptive-network-based fuzzy inference systems (ANFIS), desirability function, and genetic algorithms (GAs) to simultaneously optimize multiple correlated responses where the relationships between responses and design variables are highly nonlinear.

In the above studies, estimation of the functional relationship between control factors and responses usually relied on the accuracy of second-order polynomials, which are not always suitable [15]. Decision-makers must specify the relative weight (importance) of each response and/or set the coefficient that manipulates the shape of a desirability function subjectively while transforming multiple responses into a single objective. Furthermore, the manufacturing or material cost of a product is not considered when the optimal settings of design/process parameters are determined. To overcome these shortcomings, this study attempted to apply gene expression programming (GEP), Taguchi quality loss, and particle swarm optimization (PSO) to developing a procedure for resolving multiresponse parameter design problems. Specifically, GEP that can automatically create computer programs [19] is used to construct sophisticated nonlinear models, which are not always second-order polynomials, for estimating the functional dependence of quality characteristics (responses) on the control factors. The overall quality of a product is then evaluated through a single objective, which is the sum of the actual material cost regarding the product and the visualized Taguchi quality loss (cost) incurred owing to the deviation of each quality characteristic from its target. Notably, the quality loss coefficient can be definitely set by considering the actual material cost for fabricating the product; therefore, decision-makers no longer need to determine the relative weight of each response subjectively. 
Finally, the PSO algorithm, which has successfully resolved various optimization problems in various fields [e.g., 20-22] is utilized to determine the near optimal parameter settings of control factors by exploration of well-constructed GEP models.

The remainder of this paper is organized as follows. The fundamental methodologies utilized in this study, including the GEP and PSO, are briefly introduced in Section 2. Section 3 presents the proposed cost-based procedure for resolving multi-response parameter design problems; the feasibility and effectiveness are illustrated in Section 4 by a case study for improving the thermal performance and minimizing the material cost of a heat sink used in a high-power MR16 LED lamp. Finally, the conclusions and limitations are summarized in Section 5.

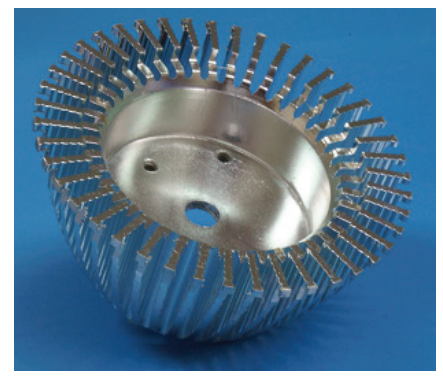

Fig. 1. Typical heat sink for a high-power MR16 LED lamp.

\section{Fundamental Methodologies}

Three main methodologies for developing the proposed procedure to resolve multi-response parameter design problems are briefly introduced in this section.

\subsection{Gene expression programming}

Gene expression programming (GEP) [23] is a technique for evolving populations of computer programs in order to solve a user-defined problem based on the principles of Darwinian natural selection and biologically inspired operations. Similar to genetic algorithms (GAs) and genetic programming (GP), GEP utilizes populations of individuals, selects them based on their fitness, and introduces genetic variation through one or more genetic operators [24]; however, there is a major difference residing in the nature of individuals. The individuals (chromosomes) in GAs are linear binary or real number strings of fixed length, and the evolved solutions in GP are usually represented by tree-based structures whose sizes and shapes can vary throughout the evolving process. However, the individuals in GEP are encoded by linear strings of fixed length (genome or chromosomes) and consist of one or more genes of equal length, which can be translated into nonlinear entities of different sizes and shapes (expression trees). The GEP gene comprises a head that contains symbols for representing both functions (elements from the function set $F$ ) and terminals (elements from the terminal set $T$ ) and a tail, which only contains terminals. For example, consider a gene composed of $[\mathrm{Q}, \times, \div,-$, $+, \mathrm{a}, \mathrm{b}]$ as follows:

$$
\begin{array}{r}
012345678901234567890 \\
-Q+\times \mathbf{b} \div \mathbf{a} \mathbf{b} \text { a Q b a b b a ba a a b b }
\end{array}
$$

where the tail is shown in bold and Q represents the square root function. Let $\alpha$ represent the maxima of the arguments for the functions considered in a gene. Since $\mathrm{Q}$ is a function of one argument, and $\times, \div,-$ and + are the functions of two arguments, the $\alpha$ corresponding to Eq. (1) is 2. In addition, the length of head $h$ is 10 (i.e., from positions 0 to 9). Hence, the length of tail $l$ can be determined to be 11 based on the following equation [23]:

$$
l=h(\alpha-1)+1=10(2-1)+1=11 .
$$

The above gene (genotype) can then be decoded and represented by an expression tree (phenotype), as shown in Fig. 2; this can easily be translated into the following algebraic expression:

$$
\sqrt{a b}-[b+a \div \sqrt{b}] .
$$

To implement a GEP procedure, some basic genetic operators including mutation, transposition, and recombination are required to perform genetic variation. Mutation can occur anywhere in a chromosome as long as the structural organization of chromosomes is intact. Hence, any symbol in the heads can change into a function or terminal while the terminals in the tails can only change into terminals. The transposition operators move fragments of the genome, which can be activated, to another place in the chromosome. Ferreira [23] defines three types of transposition in GEP: (1) insertion sequence (IS) transposition, (2) root insertion sequence (RIS) transposition, and (3) gene transposition. There are three types of recombination: (1) one-point, (2) twopoint, and (3) gene recombination. The general execution steps of GEP are briefly summarized as follows [23]: 
Step 1: Randomly generate an initial population of chromosomes.

Step 2: Express the chromosomes and evaluate the fitness of each individual.

Step 3: Select chromosomes from the population using a probability based on the fitness and replicate the selected chromosomes.

Step 4: Randomly apply genetic operators including mutation, IS transposition, RIS transposition, gene transposition, one-point recombination, two-point recombination, and gene recombination to chromosomes that are randomly selected from the replicated chromosomes in Step 3 to create the next generation.

Step 5: The outcome is designated as the final result of the run when the termination criterion is satisfied. Otherwise, iterate Steps 2-4.

As shown in the literature, GEP has produced many

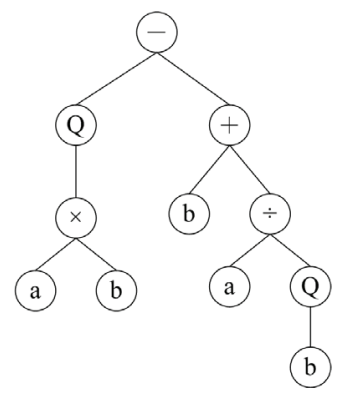

Fig. 2. Example expression tree in GEP. (Q represents the square root function.)

novel and outstanding results in resolving problems from numerous fields [e.g., 25-28]. GEP, its applications, and related resources are further discussed by $[19,23]$.

\subsection{Particle swarm optimization}

Particle swarm optimization (PSO) $[29,30]$ is a population-based stochastic optimization technique that is a form of swarm intelligence inspired by the social behavior of bird flocks, fish schools, bee swarms, and even humans. Similar to GAs, PSO initializes with a population of random solutions and searches for the optimal solution by updating generations; however, it does not have evolutionary operators such as crossover and mutation like GAs. To resolve an optimization problem, PSO works with a population of solutions called particles, whose positions and velocities are denoted by $\mathbf{x}_{i}$ and $\mathbf{v}_{i}$ ( $i$ is the index of the particle), respectively. During the search procedure, each particle keeps the best solution (position) it has already achieved, denoted by $\mathbf{x}_{i}^{\#}$, and tracks the optimal position found so far by the entire swarm, denoted by $\mathbf{x}^{*}$. The fitness values corresponding to $\mathbf{x}_{i}^{\#}$ and $\mathbf{x}^{*}$ are called pbest and gbest, respectively. Each particle then moves around in the search space toward its pbest and gbest positions with a new velocity $\mathbf{v}_{i}$, which is randomly updated based on $\mathbf{v}_{i}, \mathbf{x}_{i}, \mathbf{x}_{i}^{\#}$, and $\mathbf{x}^{*}$ in the last search iteration. A search iteration is completed when all particles have changed their velocities and positions once. The particles keep flying until they reach sufficient fitness, the pre-determined maximum number of iterations, or the pre-determined number of iterations without improvement. The general steps of PSO are briefly summarized as follows:

Step 1: A population of particles is given random positions $\mathbf{x}_{i}$ and velocities $\mathbf{v}_{i}$.

Step 2: For each particle, evaluate its fitness value and update the current local best solution $\mathbf{x}_{i}^{\#}$ and corresponding fitness pbest.

Step 3: Compare the fitness values pbests of particles in the entire swam, and update the current global best solution $\mathbf{x}^{*}$ and corresponding fitness gbest.

Step 4: Move each particle to a new position:

Step 4-1: Change the velocity of the $i$-th particle in the $j$ th dimension based on

$$
\begin{aligned}
& v_{i j}(t+1)=w v_{i j}(t)+c_{1} r_{1}\left(x_{i j}^{\#}(t)\right. \\
& \left.-x_{i j}(t)\right)+c_{2} r_{2}\left(x_{j}^{*}(t)-x_{i j}(t)\right)
\end{aligned}
$$

where $t$ is the search iteration number, $w(>0)$ is the inertia factor, $c_{1}(>0)$ and $c_{2}(>0)$ are acceleration constants, and $r_{1}$ and $r_{2}$ are random numbers uniformly distributed in the interval $[0,1]$.

Step 4-2: Change the position of each particle by

$$
x_{i j}(t+1)=x_{i j}(t)+v_{i j}(t+1)
$$

Step 5: Repeat Steps 2-4 until the stopping criteria are satisfied.

The parameter $w$ in Eq. (4), which is employed to control the impact of the previous history of velocities on the current one, is critical for the convergence behavior of PSO. A suitable value of $w$ can usually give a better tradeoff between the global and local exploration abilities of the swarm and thus reduce the number of iterations required to find the optimum 
solution. Abraham et al. [31] suggested that an initial value of around 1.2 that is gradually reduced towards 0 is a good choice for $w$. Next, the parameters $c_{1}$ and $c_{2}$, which are called the coefficients of self-recognition and social components, respectively, are stochastic acceleration terms that guide a particle where to move next by considering its best past position and position of the most successful particle in the entire swarm. Although $c_{1}$ and $c_{2}$ are not critical for the convergence of $\mathrm{PSO}$, proper settings may attain faster convergence and mitigate the local optimum [31]. Eberhart and Shi [32] recommend that settings of $c_{1}=2$ and $c_{2}=2$ are appropriate for almost all applications, and Clerc and Kennedy [33] reported that it may be better to choose a larger $c_{1}$ than $c_{2}$, but with $c_{1}+c_{2} \leq 4$. Finally, the velocity (moving distance) during one iteration $v_{i j}(t+1)$ must be clamped in the range $\left[-v_{\max }, v_{\max }\right]$ to guide the particles effectively in the search space according to

$$
v_{i j}(t+1)=\operatorname{sign}\left(v_{i j}(t+1)\right) \min \left(\left|v_{i j}(t+1)\right|, v_{\max }\right) .
$$

The parameter $v_{\max }$, which determines the solution resolution, must be carefully specified. An excessively large $v_{\max }$ may allow a particle to fly too fast and thus cause it to miss the good positions. On the other hand, a too small $v_{\max }$ may trap particles in a local optimum.

PSO has been widely applied to various optimization problems and has obtained successful results [e.g., 22, 34-36]. For further discussions on PSO, its applications, and related resources, readers can refer to $[29,37,38]$.

\section{Proposed Cost-based Solution Procedure}

An integrated procedure is proposed that resolves multiresponse parameter design problems using GEP, Taguchi quality loss, and the PSO algorithm to overcome shortcomings in the literature: insufficient accuracy of second-order polynomials, subjective determination of relative weights and shape coefficients, and non-consideration of manufacturing or material costs. As noted in Section 1, the actual material cost and visualized Taguchi quality loss due to deviations in quality characteristics from their targets are utilized to design the objective function for evaluating the overall quality of a product. For the quality characteristic $y_{j}$, the quality loss in the nominal-the-best (NTB), smallerthe-better (STB), and larger-the-better (LTB) cases are defined as

$$
\begin{gathered}
Q L_{j}=k_{j}\left(y_{j}-m_{j}\right)^{2}, \\
Q L_{j}=k_{j} y_{j}^{2},
\end{gathered}
$$

and

$$
Q L_{j}=k_{j} \frac{1}{y_{j}^{2}},
$$

respectively, where $m_{j}$ is the target value and $k_{j}$ is the quality loss coefficient for the quality characteristic $y_{j}$. Therefore, the effects of noise factors on the quality characteristic $y_{j}$ under a certain combination of control factor settings can be assessed through an average quality loss, which is calculated by

$$
A Q L_{j}=\sum_{k=1}^{s} \frac{Q L_{j}^{(k)}}{s}
$$

where $s$ is the total number of combinations of noise factor evaluation levels and $Q L_{j}^{(k)}$, which is obtained through Eq. (7), (8), or (9), is the quality loss under a certain combination of control factor settings and $k^{\text {th }}$ combination of noise factor evaluation levels. For example, there are four noise factors, and each noise factor has three evaluation levels. The noise effects on quality characteristics under a certain combination of control factor settings is appraised through 81 (i.e., $s=3^{4}$ ) combinations of noise factor evaluation levels. Finally, the overall quality regarding the product under a certain combination of control factor settings can then be evaluated through a single minimized objective function; this is calculated by

$$
T Q L=M C+\sum_{j=1}^{r} A Q L_{j}
$$

where $M C$ is the actual manufacturing or material cost of a product and $r$ is the total number of quality characteristics. The quality loss coefficients in Eqs. (7)(9) can be definitively calculated through

$$
k_{j}= \begin{cases}\frac{M C}{\left(U S L_{j}-m_{j}\right)^{2}} & \text { if } y_{j} \geq m_{j} \\ \frac{M C}{\left(L S L_{j}-m_{j}\right)^{2}} & \text { otherwise }\end{cases}
$$

for an NTB case, 


$$
\begin{gathered}
T Q L=M C+\sum_{j=1}^{r} A Q L_{j} \text { for an STB case, and (13) } \\
T Q L=M C+\sum_{j=1}^{r} A Q L_{j} \text { for an LTB case, }
\end{gathered}
$$

where $m_{j}, U S L_{j}$, and $L S L_{j}$ are the target value, upper specification limit, and lower specification limit, respectively, of the quality characteristic $y_{j}$. Since the optimal settings of control factors are determined by minimizing the sum of the actual material cost and visualized Taguchi quality loss regarding a product, the proposed approach for resolving multi-response parameter design problems is called the cost-based solution procedure. The details of the procedure are as follows:

Step 1: Determine the key quality characteristics (responses) of a product and their specification limits according to the objectives of a quality improvement project.

Step 2: Identify and arrange control factors.

Step 2-1: Identify the major design parameters (control factors) that might significantly affect the selected key quality characteristics and their design limits based on engineering principles, experience, limitations of the manufacturing process, etc.

Step 2-2:Determine the experimental levels for each selected control factor and arrange the control factors into an appropriate orthogonal array as the inner array.

Step 3: Identify and arrange noise factors.

Step 3-1:Identify the critical noise factors to be evaluated for their effects on the key quality characteristics.

Step 3-2: Determine the evaluation levels for each selected noise factor and arrange the noise factors into an appropriate orthogonal array as the outer array.

Step 4: Conduct each experimental trial and collect data according to the inner and outer arrays.

Step 5: Build GEP estimation models.

Step 5-1: Normalize the obtained values of key quality characteristics along with the values of the major design parameters into $[-1,1]$ according to their corresponding maximum and minimum values.
Step 5-2:Randomly partition the normalized values of key quality characteristics and design parameters into training and test datasets based on a pre-specified proportion.

Step 5-3: Construct an estimation model for each key quality characteristic using GEP.

Step 6: Optimize settings of control factors.

Step 6-1:Determine the optimal settings of parameters in PSO using the experimental design technique.

Step 6-2: Explore the experimental ranges of the major design parameters through PSO with the goal of minimizing the objective function defined in Eq. (11), where the mathematical relationships between the key quality characteristics and design parameters are described by the GEP models constructed in Step 5-3.

Step 6-3: Obtain the (near) optimal settings of major design parameters.

Step 7: Confirmation.

Step 7-1: Verify the feasibility and effectiveness of the optimal settings of major design parameters acquired in Step 6-3.

Step 7-2: Review the results, re-identify the control factors, and repeat Steps 2-7 if the confirmation result is unsatisfactory.

\section{Case Study}

In order to illustrate the proposed solution procedure for tackling multi-response parameter design problems, this section presents a case study that aimed to improve the design of a heat sink and thus improve its thermal performance.

\subsection{Problem statement}

In electronic systems, a heat sink is a passive heat exchanger component that cools a device such as a CPU (central processing unit), power transistor, laser, and LED by dissipating heat into the surrounding cooling medium, such as air, water, or oil. A heat sink is requisite for a device whose heat dissipation ability is insufficient to control the temperature of the basic package. Otherwise, the performance and lifetime of the device will be diminished substantially. Fig. 1 illustrates a typical heat sink applied to a high-power MR16 LED lamp. 
In order to maximize the thermal performance of a heat sink, the geometric appearance requires an elaborate design, and the material should be selected carefully. In the past, design engineers usually employed a traditional experimental design technique and the Taguchi method along with heat transfer principles and their experience to determine the optimal geometric design and select the materials of the heat sink. Due to the tradeoffs required to subjectively optimize each quality characteristic simultaneously, the final design and material selection for a heat sink were determined through a repeated trial-and-error and finetuning process. This design approach is costly, timeconsuming, and cannot ensure that the selected design and material for the heat sink are truly optimal. Furthermore, the geometric design affects the volume of the heat sink and thus determines its related material cost. Therefore, maximizing the thermal performance while minimizing the material cost of the heat sink is a crucial issue to the design of a high-power LED lamp.

\subsection{Quality characteristics and control factors}

Based on the quality improvement objectives stated in Section 4.1, designers and quality managers of LED lamps determine three key quality characteristics for a heat sink:

(1) Maximum temperature $\left(y_{1}\right)$

The maximum temperature is the stable temperature of a heat sink, as illustrated in Fig. 3, when an LED lamp is lighting. The maximum temperature is an STB-type quality characteristic.

(2) Thermal resistance $\left(y_{2}\right)$

The thermal resistance is the difference between the LED junction temperature and case temperature across a heat sink when a unit of heat energy flows through the heat sink in unit time when the lighted LED lamp has reached stable status. The thermal resistance is an STBtype quality characteristic.

(3) Material cost $\left(y_{3}\right)$

The material cost is the product of the volume, density, and unit cost of the material for fabricating a heat sink. It is an STB-type quality characteristic.

For a heat sink applied in a high-power MR16 LED lamp, the typical upper specification limits for the maximum temperature $\left(y_{1}\right)$ and thermal resistance $\left(y_{2}\right)$ are $85^{\circ} \mathrm{C}$ and $3.5^{\circ} \mathrm{C} / \mathrm{W}$, respectively, at an ambient air temperature of $25^{\circ} \mathrm{C}$.
Based on the principles of thermal physics and experience, a brainstorm with design engineers identified two critical material properties and four main geometric parameters that might significantly affect the three key quality characteristics of a heat sink of concern; these control factors are described as follows:

(1) Coefficient of thermal radiation $\left(x_{1}\right)$ : coefficient of thermal radiation of the material used to fabricate a heat sink.

(2) Coefficient of thermal conductivity $\left(x_{2}\right)$ : coefficient of thermal conductivity of the material used to fabricate a heat sink.

(3) Rotation angle $\left(x_{3}\right)$ : angle by which each fin rotates.

(4) Height of a fin $\left(x_{4}\right)$ : height of a fin in a heat sink.

(5) Width of a fin $\left(x_{5}\right)$ : width of a fin in a heat sink.

(6) Number of fins $\left(x_{6}\right)$ : total number of fins in a heat sink.

The parameters $x_{3}, x_{4}$, and $x_{5}$ are illustrated in Fig. 4 . Notably, three types of materials with different coefficients of thermal conductivity were applied in this study; their information is summarized in Table 1. In addition, two types of coefficients of thermal conductivity were considered in the case study. For each of the geometric design parameters $x_{3}$ to $x_{6}$, three experimental levels were set in order to estimate their nonlinear effects upon the critical quality characteristics. The experimental levels are summarized in Table 2. A Taguchi $L_{18}\left(2^{1} \times 3^{7}\right)$ orthogonal array was selected as the inner array, where the first control factor $\left(x_{1}\right)$ was assigned into the first column, while the remaining five design parameters (i.e., $x_{2}$ to $x_{6}$ ) were allocated into the second to sixth columns for the experiment design.

Owing to the limitations of machining while making a heat sink, the geometric parameters, including the height and width of a fin (i.e., $x_{4}$ and $x_{5}$ ) had manufacturing tolerances of $\pm 0.05 \mathrm{~mm}$. Therefore, two noise factors $z_{1}$ and $z_{2}$, each of which had three experimental settings (i.e. $-0.05,0$, and +0.05 ), were considered to assess the effects of manufacturing

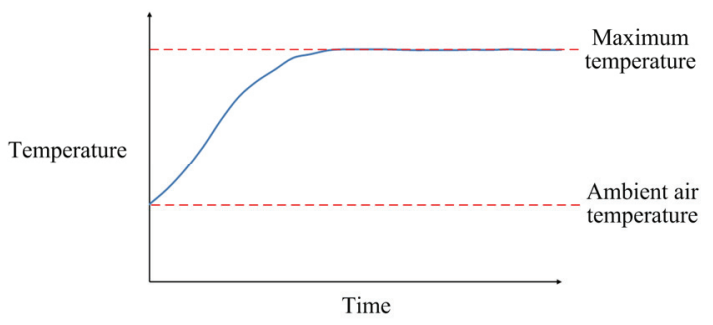

Fig. 3. Illustration of the maximum temperature. 


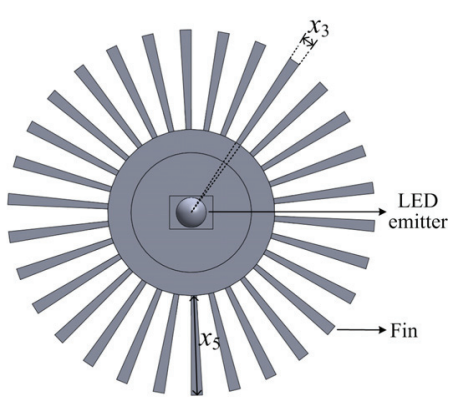

(A) Design parameters $x_{3}$ and $x_{5}$.

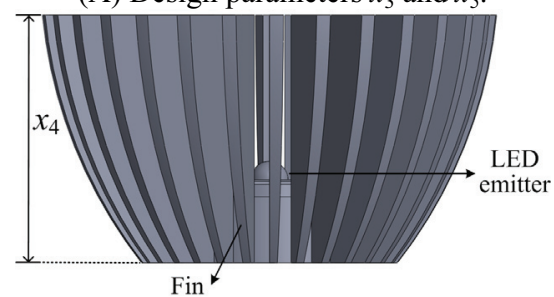

(B) Design parameter $x_{4}$.

Fig. 4. Illustration of geometric design parameters of a heat sink.

tolerances on the design parameters $x_{4}$ and $x_{5}$, respectively, as shown in Table 2 . The noise factors $z_{1}$ and $z_{2}$ were allocated in an outer array designed by a Taguchi $L_{9}\left(3^{4}\right)$ orthogonal array.

Table 1. Information about the three types of materials considered in this study.

\begin{tabular}{|c|c|c|c|}
\hline Metal & $\begin{array}{c}\text { Coefficient of thermal } \\
\text { conductivity }(\mathrm{W} / \mathrm{m} \cdot \mathrm{K})\end{array}$ & $\begin{array}{c}\text { Density } \\
\left(\mathrm{g} / \mathrm{cm}^{3}\right)\end{array}$ & Unit cost \\
\hline \hline $\mathrm{Al}$ alloy & 170 & 2.7 & 120 NTD $/ \mathrm{kg}$ \\
\hline $\mathrm{Al}$ alloy & 200 & 2.8 & $135 \mathrm{NTD} / \mathrm{kg}$ \\
\hline $\mathrm{Cu}$ & 380 & 8.9 & $288 \mathrm{NTD} / \mathrm{kg}$ \\
\hline
\end{tabular}

Table 2. Experimental settings of control and noise factors.

\begin{tabular}{|c|c|c|c|c|c|c|}
\hline $\begin{array}{c}\text { Control } \\
\text { factors }\end{array}$ & $x_{1}$ & $\begin{array}{c}x_{2} \\
(\mathrm{~W} / \mathrm{m} \cdot \mathrm{K})\end{array}$ & $\begin{array}{c}x_{3} \\
(\text { degree })\end{array}$ & $x_{4}(\mathrm{~mm})$ & $x_{5}(\mathrm{~mm})$ & $x_{6}$ \\
\hline \hline Level 1 & 0.5 & 170 & 1.5 & 18 & 10.0 & 20 \\
\hline Level 2 & 0.9 & 200 & 2.5 & 24 & 12.5 & 25 \\
\hline Level 3 & - & 380 & 3.5 & 30 & 15.0 & 30 \\
\hline $\begin{array}{c}\text { Noise } \\
\text { factors }\end{array}$ & - & - & - & $z_{1}(\mathrm{~mm})$ & $z_{2}(\mathrm{~mm})$ & - \\
\hline \hline Level 1 & - & - & - & -0.05 & -0.05 & - \\
\hline Level 2 & - & - & - & 0.00 & 0.00 & - \\
\hline Level 3 & - & - & - & +0.05 & +0.05 & - \\
\hline
\end{tabular}

\subsection{Experiments and data collection}

For this case study, 162 (i.e., $L_{18} \times L_{9}$ ) experimental trials were performed, as partially illustrated in Table 3 .
For the experiments, the SolidWorks 2010 (http://www.solidworks.com) modeling software was first used to construct the geometric model of a heat sink based on the settings for the geometric design parameters $x_{3}$ to $x_{6}$ given in Table 3. Next, the ANSYS 13 (http://www.ansys.com) software and constructed SolidWorks model were utilized to carry out the thermal simulation according to the settings for the coefficients of thermal conductivity $\left(x_{1}\right)$ and thermal radiation $\left(x_{2}\right)$, where the ambient air temperature was set to $25^{\circ} \mathrm{C}$. The LED emitter (heat source) used in this study comprised nine chips; each chip was a square with an edge length of $0.61 \mathrm{~mm}$ and thickness of $0.15 \mathrm{~mm}$. The heat energy transferred through the surface of each chip (i.e., heat flux) was set to $1.1 \mathrm{~W} / \mathrm{mm}^{2}$. The spacing between two adjacent chips and diameter of the optical lens were 0.5 and $4.5 \mathrm{~mm}$, respectively. The thickness of the base layer was set to $0.17 \mathrm{~mm}$; the substrate was a rectangular solid with a length, width, and height of 6.5, 5.0 , and $0.4 \mathrm{~mm}$, respectively. These geometric parameters are illustrated in Fig. 5. Mesh generation is a critical aspect in ANSYS simulation. Too many cells may result in long solver runs, and too few may lead to inaccurate results. Therefore, a preliminary experiment on primary sizing parameters in mesh generation, including the "relevance center" and "smoothing," was carried out based on the original heat sink design; the simulation results are summarized in Table 4. The minimum and maximum temperatures in the table are the lowest and highest temperatures, respectively, of the heat sink; the average temperature is the mean of temperatures measured at the four measurement points (MPs) located on the edge of the heat sink body, as shown in Fig. 6. The error terms (in percentage) in the last three columns are the differences between the temperatures acquired under a certain total number of nodes and their corresponding temperatures obtained with the maximum total number of nodes (i.e., $1,791,138$ nodes). As shown in Table 4, the minimum temperatures obtained with 418,203, 1,787,000, and $1,787,888$ nodes differed from the minimum temperature measured with $1,791,138$ nodes by less than $0.2 \%$; thus, the stability was considered to be sufficient. This was also true for the maximum and average temperatures. Therefore, both major sizing parameters in mesh generation (relevance center and "smoothing) were set to "medium" in this study. Table 3 presents part of the collected experimental results. 
Notably, the thermal resistance $\left(y_{2}\right)$ was calculated based on the maximum temperature $\left(y_{1}\right)$ and average temperature. The material cost $\left(y_{3}\right)$ was determined based on the volume, density and unit cost of the material; the density and unit cost of the material depended on the coefficient of thermal conductivity $\left(x_{2}\right)$, as described in Section 4.2. Therefore, the average temperatures and volumes, which were treated as dependent variables during construction of the GEP estimation models presented later, are also given in Table 3.

Table 3. Partial experimental trials and collected data.

\begin{tabular}{|c|c|c|c|c|c|c|c|c|c|c|c|c|c|}
\hline \multirow[b]{2}{*}{ No. } & \multicolumn{6}{|c|}{ Control factors } & \multicolumn{2}{|c|}{ Noise factors } & \multicolumn{3}{|c|}{ Quality characteristics } & \multirow[b]{2}{*}{$\begin{array}{c}\text { Average } \\
\text { temperature } \\
\left({ }^{\circ} \mathrm{C}\right)\end{array}$} & \multirow[b]{2}{*}{$\begin{array}{l}\text { Volume } \\
\left(\mathrm{mm}^{3}\right)\end{array}$} \\
\hline & $x_{1}$ & $\begin{array}{c}x_{2} \\
(\mathrm{~W} / \mathrm{m} \cdot \mathrm{K})\end{array}$ & $\begin{array}{c}x_{3} \\
\text { (degree) }\end{array}$ & $\begin{array}{c}x_{4} \\
(\mathrm{~mm})\end{array}$ & $\begin{array}{c}x_{5} \\
(\mathrm{~mm})\end{array}$ & $x_{6}$ & $\begin{array}{c}z_{1} \\
(\mathrm{~mm})\end{array}$ & $\begin{array}{c}z_{2} \\
(\mathrm{~mm})\end{array}$ & \begin{tabular}{|c|} 
Maximum \\
temperature \\
$\left(y_{1},{ }^{\circ} \mathrm{C}\right)$ \\
\end{tabular} & $\begin{array}{c}\text { Thermal } \\
\text { resistance } \\
\left(y_{2},{ }^{\circ} \mathrm{C} / \text { Watt }\right)\end{array}$ & $\begin{array}{c}\text { Material } \\
\text { cost } \\
\left(y_{3}, \mathrm{NTD}\right)\end{array}$ & & \\
\hline 1 & 0.5 & 170 & 1.5 & 18 & 10.0 & 20 & -0.05 & -0.05 & 69.27 & 3.5774 & 0.6686 & 55.74 & 2064 \\
\hline 2 & 0.5 & 170 & 1.5 & 18 & 10.0 & 20 & -0.05 & 0.00 & 69.14 & 3.5804 & 0.6713 & 55.60 & 2072 \\
\hline 3 & 0.5 & 170 & 1.5 & 18 & 10.0 & 20 & -0.05 & +0.05 & 69.01 & 3.5884 & 0.6740 & 55.45 & 2080 \\
\hline 4 & 0.5 & 170 & 1.5 & 18 & 10.0 & 20 & 0.00 & -0.05 & 69.13 & 3.5857 & 0.6819 & 55.58 & 2105 \\
\hline 5 & 0.5 & 170 & 1.5 & 18 & 10.0 & 20 & 0.00 & 0.00 & 69.00 & 3.5860 & 0.6848 & 55.45 & 2113 \\
\hline 6 & 0.5 & 170 & 1.5 & 18 & 10.0 & 20 & 0.00 & +0.05 & 69.09 & 3.5940 & 0.6825 & 55.50 & 2107 \\
\hline 7 & 0.5 & 170 & 1.5 & 18 & 10.0 & 20 & +0.05 & -0.05 & 68.99 & 3.6047 & 0.6954 & 55.37 & 2146 \\
\hline 8 & 0.5 & 170 & 1.5 & 18 & 10.0 & 20 & +0.05 & 0.00 & 69.08 & 3.5818 & 0.6931 & 55.54 & 2139 \\
\hline 9 & 0.5 & 170 & 1.5 & 18 & 10.0 & 20 & +0.05 & +0.05 & 68.95 & 3.6036 & 0.6960 & 55.33 & 2148 \\
\hline \\
\hline 154 & 0.9 & 380 & 3.5 & 24 & 10.0 & 25 & -0.05 & -0.05 & 55.19 & 3.1507 & 13.9139 & 43.28 & 5428 \\
\hline 155 & 0.9 & 380 & 3.5 & 24 & 10.0 & 25 & -0.05 & 0.00 & 55.12 & 3.1478 & 13.9959 & 43.22 & 5460 \\
\hline 156 & 0.9 & 380 & 3.5 & 24 & 10.0 & 25 & -0.05 & +0.05 & 55.04 & 3.1608 & 14.0782 & 43.10 & 5492 \\
\hline 157 & 0.9 & 380 & 3.5 & 24 & 10.0 & 25 & 0.00 & -0.05 & 55.12 & 3.1575 & 14.1302 & 43.19 & 5513 \\
\hline 158 & 0.9 & 380 & 3.5 & 24 & 10.0 & 25 & 0.00 & 0.00 & 55.05 & 3.1381 & 14.2135 & 43.19 & 5545 \\
\hline 159 & 0.9 & 380 & 3.5 & 24 & 10.0 & 25 & 0.00 & +0.05 & 55.11 & 3.1380 & 14.1390 & 43.24 & 5516 \\
\hline 160 & 0.9 & 380 & 3.5 & 24 & 10.0 & 25 & +0.05 & -0.05 & 55.05 & 3.1633 & 14.3480 & 43.10 & 5598 \\
\hline 161 & 0.9 & 380 & 3.5 & 24 & 10.0 & 25 & +0.05 & 0.00 & 55.11 & 3.1585 & 14.2726 & 43.17 & 5568 \\
\hline 162 & 0.9 & 380 & 3.5 & 24 & 10.0 & 25 & +0.05 & +0.05 & 55.04 & 3.1513 & 14.3571 & 43.12 & 5601 \\
\hline
\end{tabular}

Table 4. Results of a preliminary experiment on primary sizing parameters.

\begin{tabular}{|c|c|c|c|c|c|c|c|c|}
\hline $\begin{array}{c}\text { Relevance } \\
\text { center }\end{array}$ & Smoothing & Nodes & $\begin{array}{c}\text { Minimum } \\
\text { temperature } \\
\left({ }^{\circ} \mathrm{C}\right)\end{array}$ & $\begin{array}{c}\text { Maximum } \\
\text { temperature } \\
\left({ }^{\circ} \mathrm{C}\right)\end{array}$ & $\begin{array}{c}\text { Average } \\
\text { temperature } \\
\left({ }^{\circ} \mathrm{C}\right)\end{array}$ & $\begin{array}{c}\text { Error of } \\
\text { minimum } \\
\text { temperature }\end{array}$ & $\begin{array}{c}\text { Error of } \\
\text { maximum } \\
\text { temperature }\end{array}$ & $\begin{array}{c}\text { Error of } \\
\text { average } \\
\text { temperature }\end{array}$ \\
\hline \hline coarse & low & 68,359 & 52.25 & 38.31 & 39.71 & $1.5507 \%$ & $0.0078 \%$ & $0.2694 \%$ \\
\hline coarse & high & 68,374 & 52.32 & 38.31 & 39.73 & $1.4207 \%$ & $0.0078 \%$ & $0.2009 \%$ \\
\hline coarse & medium & 68,565 & 52.32 & 38.31 & 39.71 & $1.4188 \%$ & $0.0078 \%$ & $0.2587 \%$ \\
\hline medium & high & 417,945 & 53.16 & 38.31 & 39.76 & $0.1620 \%$ & $0.0052 \%$ & $0.1457 \%$ \\
\hline medium & low & 417,949 & 53.21 & 38.32 & 39.76 & $0.2619 \%$ & $0.0026 \%$ & $0.1369 \%$ \\
\hline medium & medium & 418,203 & 53.16 & 38.31 & 39.76 & $0.1620 \%$ & $0.0052 \%$ & $0.1425 \%$ \\
\hline fine & high & $1,787,000$ & 53.07 & 38.32 & 39.82 & $0.0019 \%$ & $0.0026 \%$ & $0.0019 \%$ \\
\hline fine & medium & $1,787,888$ & 53.07 & 38.32 & 39.81 & $0.0019 \%$ & $0.0026 \%$ & $0.0201 \%$ \\
\hline fine & low & $1,791,138$ & 53.07 & 38.32 & 39.81 & - & & - \\
\hline
\end{tabular}




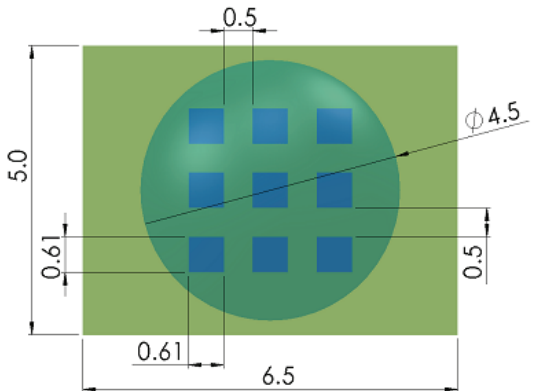

(A) Top view of the LED emitter.

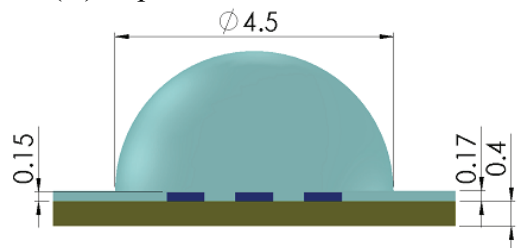

(B) Side view of the LED emitter.

Fig. 5. Major geometric parameters of the LED emitter used in this study.

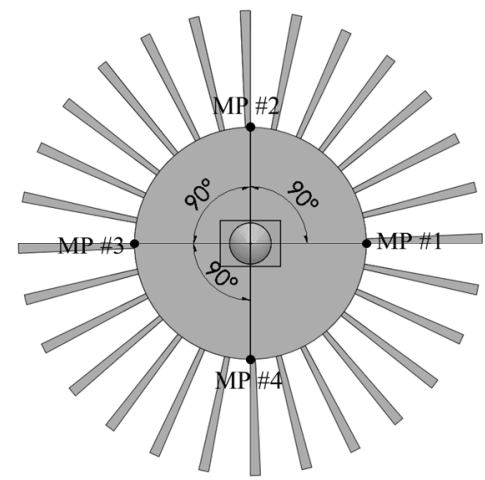

Fig. 6. Measurement points for assessing the average temperature.

\subsection{Building GEP estimation models}

The maximum temperature, average temperature, and volume along with the six design parameters obtained in each experimental trial are partially shown in Table 3; they were normalized into the range of -1 to 1 according to their corresponding maximum and minimum values and then randomly partitioned into training and test datasets at a proportion of $4: 1$. The GEP algorithm using the GeneXproTools 4.0 (http://www.gepsoft.com) software was employed on the training and test datasets to build the mathematical models for estimating the dependence of the maximum temperature, average temperature, and volume on the design parameters. The fitness of an individual was evaluated through root relative squared error (RRSE). In addition, the parameters, function set and terminal set in GEP were set as their default values. Table 5 summarizes the settings of the major parameters in GEP. For each of the maximum temperature, average temperature and volume, the GEP algorithm was implemented for 5 runs. The results are summarized in Table 6. Based on the principle of minimizing the training and test RRSEs simultaneously, the second, first, and fifth models in Table 6 (i.e., GEP_ $\mathrm{M}_{\mathrm{mt}}$, GEP_M $M_{a t}$, and GEP_ $M_{v o}$, respectively) were selected to estimate the maximum temperature, average temperature, and volume, respectively, for a given combination of settings for the six design parameters. Appendix A summarizes the execution programs in the $\mathrm{C}++$ language for the GEP_ $\mathrm{M}_{\mathrm{mt}}, \mathrm{GEP}_{-} \mathrm{M}_{\mathrm{at}}$, and GEP_M $\mathrm{M}_{\mathrm{vo}}$ models obtained by the GeneXproTools 4.0 software. Notably, the final expression trees, which are encoded by the function and terminal sets in GEP, are automatically determined through the evolving procedure, as described in Section 2.1. Therefore, the functions and terminals included in the final execution programs for the GEP_ $\mathrm{M}_{\mathrm{mt}}$, GEP_ $\mathrm{M}_{\mathrm{at}}$, and GEP_ $\mathrm{M}_{\mathrm{vo}}$ models are not necessarily identical.

Table 5. Settings of major parameters in GEP.

\begin{tabular}{|l|c|}
\hline \multicolumn{1}{|c|}{ Parameter } & Setting \\
\hline \hline Number of chromosomes & 30 \\
\hline Head size & 8 \\
\hline Number of genes & 3 \\
\hline Mutation rate & 0.044 \\
\hline IS transposition rate & 0.1 \\
\hline RIS transposition rate & 0.1 \\
\hline Gene transposition rate & 0.1 \\
\hline One-point recombination rate & 0.3 \\
\hline Two-point recombination rate & 0.3 \\
\hline Gene recombination rate & 0.1 \\
\hline
\end{tabular}


Table 6. Execution results of the GEP algorithm.

\begin{tabular}{|c|c|c|c|c|c|c|}
\hline $\begin{array}{c}\text { Dependent } \\
\text { variable }\end{array}$ & $\begin{array}{c}\text { Trial } \\
\text { number }\end{array}$ & $\begin{array}{c}\text { Training } \\
\text { RRSE }\end{array}$ & $\begin{array}{c}\text { Test } \\
\text { RRSE }\end{array}$ & $\begin{array}{c}\text { Training } \\
\mathrm{R}^{2}\end{array}$ & $\begin{array}{c}\text { Test } \\
\mathrm{R}^{2}\end{array}$ & $\begin{array}{c}\text { GEP } \\
\text { Model } \\
\text { name }\end{array}$ \\
\hline \multirow{5}{*}{$\begin{array}{l}\text { Maximum } \\
\text { temperature }\end{array}$} & 1 & 0.1438 & 0.2554 & 0.9797 & 0.9376 & \\
\hline & $2 *$ & 0.1539 & 0.2187 & 0.9769 & 0.9834 & GEP_M $M_{\mathrm{mt}}$ \\
\hline & 3 & 0.1539 & 0.2187 & 0.9769 & 0.9834 & \\
\hline & 4 & 0.1539 & 0.2187 & 0.9769 & 0.9834 & \\
\hline & 5 & 0.1695 & 0.3199 & 0.9729 & 0.9028 & \\
\hline \multirow{5}{*}{$\begin{array}{l}\text { Average } \\
\text { temperature }\end{array}$} & $1 *$ & 0.0832 & 0.0853 & 0.9932 & 0.9929 & GEP_M $M_{a t}$ \\
\hline & 2 & 0.0832 & 0.0853 & 0.9932 & 0.9929 & \\
\hline & 3 & 0.1358 & 0.1327 & 0.9816 & 0.9830 & \\
\hline & 4 & 0.0833 & 0.0858 & 0.9931 & 0.9928 & \\
\hline & 5 & $\begin{array}{l}0.1449 \\
\end{array}$ & 0.1740 & 0.9790 & 0.9724 & \\
\hline \multirow{5}{*}{ Volume } & 1 & 0.1126 & 0.1431 & 0.9875 & 0.9811 & \\
\hline & 2 & 0.1126 & 0.1431 & 0.9875 & 0.9811 & \\
\hline & 3 & 0.1126 & 0.1431 & 0.9875 & 0.9811 & \\
\hline & 4 & 0.1126 & 0.1431 & 0.9875 & 0.9811 & \\
\hline & $5 *$ & 0.1034 & 0.1385 & 0.9904 & 0.9811 & GEP_M ${ }_{\mathrm{vo}}$ \\
\hline
\end{tabular}

\subsection{Optimization of design parameters}

In order to determine the optimal settings of the six design parameters for a heat sink, the PSO algorithm was applied to explore the experimental ranges of the design parameters. The GEP_M $\mathrm{M}_{\mathrm{mt}}, \mathrm{GEP}_{-} \mathrm{M}_{\mathrm{at}}$, and GEP_M $\mathrm{M}_{\mathrm{vo}}$ models presented in Section 4.4 were used to describe the mathematical dependence of the maximum temperature, average temperature, and volume, respectively, on the design parameters. The objective function for minimization by PSO was designed using Eq. (11). The quality losses for the maximum temperature $\left(y_{1}\right)$ and thermal resistance $\left(y_{2}\right)$ were calculated using Eq. (8); the quality loss coefficients were derived from Eq. (13). The actual material cost of a heat sink (i.e., $M C$ in Eqs. (11) and (13)) was calculated based on the volume, density, and unit cost of the material, as shown in Table 1, depending on the coefficient of thermal conductivity $\left(x_{2}\right)$. In addition, the upper specification limits (i.e., $U S L_{j}$ in Eq. (13)) for the maximum temperature $\left(y_{1}\right)$ and thermal resistance $\left(y_{2}\right)$ were set to $85{ }^{\circ} \mathrm{C}$ and $3.5{ }^{\circ} \mathrm{C} / \mathrm{W}$, respectively, as noted in Section 4.2. In addition, $75{ }^{\circ} \mathrm{C}$ and $3.0^{\circ} \mathrm{C} / \mathrm{W}$ were considered to be sufficiently ideal values for the maximum temperature $\left(y_{1}\right)$ and thermal resistance $\left(y_{2}\right)$, respectively, based on consultation with design engineers. Therefore, the quality loss for a maximum temperature $\left(y_{1}\right)$ of less than $75^{\circ} \mathrm{C}$ or thermal resistance $\left(y_{2}\right)$ of less than $3.0^{\circ} \mathrm{C} / \mathrm{W}$ was directly set to 0 in this study.

To determine the optimal settings of major parameters in $\mathrm{PSO}$ - the inertia factor $(w)$, ratio of inertia factor $\left(r_{w}\right)$, maximum velocity $\left(v_{\max }\right)$, coefficient of the self-recognition component $\left(c_{1}\right)$, and coefficients of the social component $\left(c_{2}\right)-\mathrm{a} 2^{5-1}$ fractional factorial design was used, as shown in Table 7. This is a good choice for controlling the appropriate value of inertia factor $w$ by initially setting the factor as a constant and gradually reducing it towards 0 [31]. Hence, the inertia factor $w$ was designed to decrease progressively through multiplication with the ratio of the inertia factor $\left(r_{w}\right)$ after every 10 search cycles in this study. Design-Expert 6.0 (http://www.statease.com) software was then utilized to analyze the collected data shown in Table 7 and automatically selected the parameter $w_{r}$, interaction $w \times v_{\max }$ and interaction $c_{1} \times c_{2}$ into the ANOVA model, which was significant at $\alpha=0.05$ as shown in Table 8 . According to the effects of parameters, which are summarized in Table 9, the optimal settings of $w_{r}, w$, $v_{\max }, c_{1}$, and $c_{2}$ in PSO were set to $0.99,0.8,0.10,1$, and 1 , respectively. The population size was set to 100 , and the PSO algorithm terminated if the objective value could not be further improved after the last 100 search cycles. The PSO search procedure was implemented for 10 runs, which were executed on a personal computer with an Intel Core i7-3770 3.40 GHz CPU and $8 \mathrm{~GB}$ RAM; the optimization results are summarized in Table 10. In order to minimize the objective function, the following combination of design parameter settings, was determined to be the optimal solution: $x_{1}=0.9, x_{2}=$ $200 \mathrm{~W} / \mathrm{m} \cdot \mathrm{K}, x_{3}=3.5^{\circ}, x_{4}=20.61 \mathrm{~mm}, x_{5}=12.03 \mathrm{~mm}$, and $x_{5}=24$. This was the fifth run, as shown in Table 10 . The coefficient of variation regarding the objective function was small at 0.0351 ; the PSO completed its search procedure in less than $1 \mathrm{~s}$ of CPU time. Based on the above information, the PSO algorithm can be considered to be a robust and highly speedy optimization method for exploring an experimental range to find the (near) optimal settings of control factors in a multi-response parameter design problem. Furthermore, the coefficient of thermal conductivity of the material was determined to be $200 \mathrm{~W} / \mathrm{m} \cdot \mathrm{K}$ from the optimal combination of the design parameter settings. Notably, the coefficient of thermal conductivity of 200 $\mathrm{W} / \mathrm{m} \cdot \mathrm{K}$ was not the highest among the three types of materials considered in this study. This implies that a designer should first attempt to maximize the thermal performance of a heat sink through optimizing the geometric design, but not through making use of the material with a higher coefficient of thermal conductivity, which is much more expensive. 
Table 7. Experiment on PSO parameters.

\begin{tabular}{|c|c|c|c|c|c|c|c|c|c|c|}
\hline \multirow{2}{*}{ No. } & \multirow{2}{*}{$w$} & \multirow{2}{*}{$w_{r}$} & \multirow[b]{2}{*}{$v_{\max }$} & \multirow[b]{2}{*}{$c_{1}$} & \multirow[b]{2}{*}{$c_{-}$} & \multicolumn{5}{|c|}{ Objective function } \\
\hline & & & & & & Rep. 1 & Rep. 2 & Rep. 3 & \begin{tabular}{|l|} 
Rep. 4 \\
\end{tabular} & Rep. 5 \\
\hline 1 & 0.8 & 0.90 & 0.05 & 1 & 2 & 2.6542 & 2.9797 & 3.3826 & 3.4018 & 4.8670 \\
\hline 2 & 1.0 & 0.90 & 0.05 & 1 & 1 & 2.9183 & 2.6108 & 1.6169 & 1.6575 & 3.0617 \\
\hline 3 & 0.8 & 0.99 & 0.05 & 1 & 1 & 3.0347 & 1.9194 & 3.9395 & 2.2624 & 2.6576 \\
\hline 4 & 1.0 & 0.99 & 0.05 & 1 & 2 & 2.6494 & 1.8835 & 2.2539 & 2.6497 & 2.9183 \\
\hline 5 & 0.8 & 0.90 & 0.10 & 1 & 1 & 2.9183 & 2.0460 & 2.6493 & 2.6493 & 1.8838 \\
\hline 6 & 1.0 & 0.90 & 0.10 & 1 & 2 & 3.6146 & 2.6494 & 2.8191 & 2.8174 & 2.9183 \\
\hline 7 & 0.8 & 0.99 & 0.10 & 1 & 2 & 2.6498 & 2.6493 & 2.8235 & 1.6351 & 2.6493 \\
\hline 8 & 1.0 & 0.99 & 0.10 & 1 & 1 & 1.6349 & 1.6348 & 2.8175 & 2.7009 & 2.8174 \\
\hline 9 & 0.8 & 0.90 & 0.05 & $\frac{2}{2} \cdot \ln \cdot \mathrm{l}$ & 1 & 2.9187 & 5.9125 & 2.6918 & 5.3939 & 3.4902 \\
\hline 10 & 1.0 & 0.90 & 0.05 & 2 & 2 & 2.6536 & 2.6527 & 2.6914 & 4.5382 & 2.6898 \\
\hline 11 & 0.8 & 0.99 & 0.05 & 2 & 2 & 2.6586 & 2.6493 & 1.8835 & 1.5866 & 2.6666 \\
\hline 12 & 1.0 & 0.99 & 0.05 & 2 & 1 & 2.6493 & 2.6493 & 2.6493 & 2.6494 & 2.6493 \\
\hline 13 & 0.8 & 0.90 & 0.10 & 2 & 2 & 2.9183 & 2.8405 & 2.8187 & 2.6493 & 2.8174 \\
\hline 14 & 1.0 & 0.90 & 0.10 & 2 & 1 & 2.9651 & 1.8835 & 2.8349 & 2.9183 & 3.6146 \\
\hline 15 & 0.8 & \begin{tabular}{|l|}
0.99 \\
\end{tabular} & 0.10 & 2 & 1 & 2.8177 & 2.8174 & 2.6493 & 1.8835 & 1.6389 \\
\hline 16 & 1.0 & \begin{tabular}{|l|}
0.99 \\
\end{tabular} & 0.10 & 2 & 2 & 2.8181 & 2.8192 & 2.9183 & 2.6493 & \begin{tabular}{|l|l}
1.5559 \\
\end{tabular} \\
\hline
\end{tabular}

Table 8. ANOVA for experiment on PSO parameters.

\begin{tabular}{|c|c|c|c|c|c|}
\hline Source & $\begin{array}{c}\text { Sum of } \\
\text { squares }\end{array}$ & d.f. & $\begin{array}{c}\text { Mean } \\
\text { square }\end{array}$ & $\begin{array}{c}\mathrm{F} \\
\text { value }\end{array}$ & Significance \\
\hline Model & 9.81 & 3 & 9.81 & 6.98 & 0.0003 \\
\hline$w_{r}$ & 5.29 & 1 & 5.29 & 11.29 & 0.0012 \\
\hline$w_{r} \times v_{\max }$ & 2.20 & 1 & 2.20 & 4.69 & 0.0335 \\
\hline$c_{1} \times c_{2}$ & 2.32 & 1 & 2.32 & 4.96 & 0.0289 \\
\hline Residual & 35.61 & 76 & 0.47 & & \\
\hline $\begin{array}{l}\text { Lack of } \\
\text { Fit }\end{array}$ & 7.23 & 12 & 0.60 & 1.36 & 0.2089 \\
\hline Pure Error & 28.38 & 64 & 0.44 & & \\
\hline $\begin{array}{l}\text { Corrected } \\
\text { Total }\end{array}$ & 45.42 & 79 & & & \\
\hline
\end{tabular}

Table 9. Effects of parameters in PSO.

(A) Effect of the parameter $w_{r}$

\begin{tabular}{|c|c|}
\hline$w_{r}=0.90$ & $w_{r}=0.99^{*}$ \\
\hline \hline $\mathrm{MO}=3.0002$ & $\mathrm{MO}=2.4860$ \\
\hline
\end{tabular}

(B) Effects of the parameters $w$ and $v_{\max }$

\begin{tabular}{|l|c|c|}
\hline & $w=0.8^{*}$ & $w=1.0$ \\
\hline \hline$v_{\max }=0.05$ & $\mathrm{MO}=3.1475$ & $\mathrm{MO}=2.6346$ \\
\hline$v_{\max }=0.10^{*}$ & $\mathrm{MO}=2.5202$ & $\mathrm{MO}=2.6701$ \\
\hline
\end{tabular}

(B) Effects of the parameters $c_{1}$ and $c_{2}$

\begin{tabular}{|c|c|c|}
\hline & $c_{1}=1^{*}$ & $c_{1}=2$ \\
\hline \hline$c_{2}=1^{*}$ & $\mathrm{MO}=2.4716$ & $\mathrm{MO}=2.9839$ \\
\hline$c_{2}=2$ & $\mathrm{MO}=2.8433$ & $\mathrm{MO}=2.6738$ \\
\hline
\end{tabular}

Note: "MO" represents the mean of the objective function's values under a certain parameter's setting or a certain combination of parameter settings; the asterisk denotes the optimal setting for a parameter in PSO.

Table 10. Optimization results obtained by PSO.

\begin{tabular}{|c|c|c|c|c|c|c|c|c|c|}
\hline No. & $x_{1}$ & $\begin{array}{c}x_{2} \\
(\mathrm{~W} / \mathrm{m} \cdot \mathrm{K})\end{array}$ & $\begin{array}{c}x_{3} \\
(\mathrm{degree})\end{array}$ & $\begin{array}{c}x_{4} \\
(\mathrm{~mm})\end{array}$ & $\begin{array}{c}x_{5} \\
(\mathrm{~mm})\end{array}$ & $x_{6}$ & $\begin{array}{c}\text { Objective } \\
\text { function }\end{array}$ & $\begin{array}{c}\text { Search } \\
\text { cycle }\end{array}$ & $\begin{array}{c}\text { CPU Time } \\
(\mathrm{s})\end{array}$ \\
\hline \hline 1 & 0.9 & 170 & 3.5 & 20.41 & 11.95 & 23 & 2.8223 & 393 & 0.41 \\
\hline 2 & 0.9 & 200 & 3.5 & 20.84 & 12.33 & 23 & 2.9183 & 356 & 0.36 \\
\hline 3 & 0.9 & 170 & 3.5 & 20.54 & 11.87 & 23 & 2.8182 & 433 & 0.43 \\
\hline 4 & 0.9 & 200 & 3.5 & 20.84 & 12.33 & 23 & 2.9183 & 747 & 0.79 \\
\hline 5 & 0.9 & 200 & 3.5 & 20.61 & 12.03 & 24 & 2.6492 & 438 & 0.44 \\
\hline 6 & 0.9 & 200 & 3.5 & 20.60 & 12.03 & 24 & 2.6494 & 425 & 0.43 \\
\hline 7 & 0.9 & 170 & 3.5 & 20.30 & 11.99 & 23 & 2.8402 & 296 & 0.29 \\
\hline 8 & 0.9 & 170 & 3.5 & 20.66 & 11.77 & 23 & 2.8358 & 307 & 0.31 \\
\hline 9 & 0.9 & 200 & 3.5 & 20.84 & 12.33 & 23 & 2.9183 & 934 & 0.93 \\
\hline 10 & 0.9 & 170 & 3.5 & 20.33 & 11.98 & 23 & 2.8355 & 580 & 0.58 \\
\hline Mean & - & - & - & - & - & - & 2.8206 & 491 & 0.50 \\
\hline $\begin{array}{c}\text { Coefficient } \\
\text { of variation }\end{array}$ & - & - & - & - & - & - & 0.0351 & 0.4185 & 0.4226 \\
\hline
\end{tabular}

\subsection{Confirmation experiment}

A confirmation experiment was conducted to verify the feasibility and effectiveness of the optimal combination of design parameter settings identified in Section 4.5. The results are summarized in the first trial given in
Table 11. As noted in Section 4.2, the height of a fin $\left(x_{4}\right)$ and width of a fin $\left(x_{5}\right)$ have a manufacturing tolerance of $\pm 0.05 \mathrm{~mm}$ due to the precision limitations for a machined heat sink. Hence, two noise factors $z_{1}$ and $z_{2}$, each with three experimental settings of $-0.05,0$, and +0.05 , were considered in the case study. The effects 
of the noise factors on each quality characteristic were then assessed through the average quality loss calculated by Eq. (10), as described in Section 3, in order to find the robust design of a heat sink-i.e., design that is insensitive to the noise factors. To examine the effectiveness of considering the noise factors into the objective function for optimizing the control factors, nine extra confirmation trials were conducted, as shown by the second to tenth trials in Table 11, based on the nine possible combinations of noise factors $z_{1}$ and $z_{2}$. As noted previously, the material cost $\left(y_{3}\right)$ depends on the heat sink volume, density and unit cost of the material; the density and unit cost of the material are determined by the coefficient of thermal conductivity $\left(x_{2}\right)$. Although $\mathrm{Cu}$ metal with a coefficient of thermal conductivity of $380 \mathrm{~W} / \mathrm{m} \cdot \mathrm{K}$, as shown in Table 1, possessed the best thermal conductivity, its unit cost was also the highest among the three types of metals considered in this study. In the confirmation experiment based on the optimal settings of design parameters acquired in Section 4.5, Al alloy-with a coefficient of thermal conductivity of $200 \mathrm{~W} / \mathrm{m} \cdot \mathrm{K}$ and about $47 \%$ of the unit cost of $\mathrm{Cu}$ - was used to fabricate a heat sink. Based on the simulation results given in Table 11, the maximum temperature $\left(y_{1}\right)$ and thermal resistance $\left(y_{2}\right)$ in all confirmation trials met their specification limits of $85^{\circ} \mathrm{C}$ and $3.5^{\circ} \mathrm{C} / \mathrm{W}$, respectively.
This implies that the proposed cost-based solution procedure, which aims to minimize both the actual material cost and visualized Taguchi quality loss of a product, indeed provides an optimal design of a heat sink that maximizes the critical quality characteristics (i.e., maximum temperature $\left(y_{1}\right)$ and thermal resistance $\left.\left(y_{2}\right)\right)$ and minimizes the actual material cost by selection of the optimal material type for a geometric design determined by parameters $x_{3}$ to $x_{6}$. The coefficients of variation for the maximum temperature $\left(y_{1}\right)$, thermal resistance $\left(y_{2}\right)$, average temperature, and volume were all smaller than $8 \times 10^{-3}$; thus, the design is considered to be sufficiently robust. This indicates that assessing the noise factor effects on quality characteristics by using the average quality loss defined by (10) can indeed significantly reduce the sensitivity of a design to noise factors-i.e., increase the robustness of a heat sink's design. Based on the above results, the case study on improving the thermal performance of a heat sink by optimizing the geometric design and selecting the most appropriate material was successful. Therefore, the proposed systematic approach in this study is a feasible and effective way of resolving multi-response parameter design problems in terms of simultaneously minimizing the actual material cost and visualized Taguchi quality loss of a product.

Table 11. Summary of confirmation experiment results.

\begin{tabular}{|c|c|c|c|c|c|c|c|c|c|c|c|}
\hline No. & $x_{1}$ & $\begin{array}{c}x_{2} \\
(\mathrm{~W} / \mathrm{m} \cdot \mathrm{K})\end{array}$ & $\begin{array}{c}x_{3} \\
(\text { degree })\end{array}$ & $\begin{array}{c}x_{4} \\
(\mathrm{~mm})\end{array}$ & $\begin{array}{c}x_{5} \\
(\mathrm{~mm})\end{array}$ & $x_{6}$ & $\begin{array}{c}\text { Maximum } \\
\text { temperature }\left(y_{1},{ }^{\circ} \mathrm{C}\right)\end{array}$ & $\begin{array}{c}\text { Thermal resistance } \\
\left(y_{2},{ }^{\circ} \mathrm{C} / \text { Watt }\right)\end{array}$ & $\begin{array}{c}\text { Material cost } \\
\left(y_{3}, \mathrm{NTD}\right)\end{array}$ & $\begin{array}{c}\text { Average } \\
\text { temperature }\left({ }^{\circ} \mathrm{C}\right)\end{array}$ & $\begin{array}{c}\text { Volume } \\
\left(\mathrm{mm}^{3}\right)\end{array}$ \\
\hline 1 & 0.9 & 200 & 3.5 & 20.61 & 12.03 & 24 & 57.34 & 3.4923 & 2.0067 & 44.14 & 5309 \\
\hline 2 & 0.9 & 200 & 3.5 & 20.56 & 11.98 & 24 & 57.42 & 3.4880 & 1.9952 & 44.23 & 5278 \\
\hline 3 & 0.9 & 200 & 3.5 & 20.56 & 12.03 & 24 & 57.37 & 3.4830 & 2.0020 & 44.21 & 5296 \\
\hline 4 & 0.9 & 200 & 3.5 & 20.56 & 12.08 & 24 & 57.33 & 3.4896 & 2.0089 & 44.14 & 5315 \\
\hline 5 & 0.9 & 200 & 3.5 & 20.61 & 11.98 & 24 & 57.38 & 3.4804 & 1.9998 & 44.23 & 5290 \\
\hline 6 & 0.9 & 200 & 3.5 & 20.61 & 12.03 & 24 & 57.34 & 3.4804 & 2.0067 & 44.18 & 5309 \\
\hline 7 & 0.9 & 200 & 3.5 & 20.61 & 12.08 & 24 & 57.30 & 3.4913 & 2.0135 & 44.10 & 5327 \\
\hline 8 & 0.9 & 200 & 3.5 & 20.66 & 11.98 & 24 & 57.35 & 3.4793 & 2.0044 & 44.20 & 5303 \\
\hline 9 & 0.9 & 200 & 3.5 & 20.66 & 12.03 & 24 & 57.30 & 3.4883 & 2.0113 & 44.12 & 5321 \\
\hline 10 & 0.9 & 200 & 3.5 & 20.66 & 12.08 & 24 & 57.26 & 3.4664 & 2.0182 & 44.16 & 5339 \\
\hline Max & - & - & - & - & - & - & 57.42 & 3.4923 & 2.0182 & 44.23 & 5339 \\
\hline Min & - & - & - & - & - & - & 57.26 & 3.4664 & 1.9952 & 44.10 & 5278 \\
\hline Mean & - & - & - & - & - & - & 57.34 & 3.4839 & 2.0067 & 44.17 & 5309 \\
\hline $\begin{array}{l}\text { Coefficient } \\
\text { of variation }\end{array}$ & - & - & - & - & - & - & $7.88 \times 10^{-4}$ & $7.79 \times 10^{-3}$ & $6.78 \times 10^{-3}$ & $1.05 \times 10^{-3}$ & $3.38 \times 10^{-3}$ \\
\hline
\end{tabular}

\subsection{Analysis through Taguchi method}

The full collected experimental data, which are partially shown in Table 3, were further analyzed through the Taguchi method. Table 12 summarizes the results; the
$\mathrm{S} / \mathrm{N}$ ratios were analyzed, where the text with grey background denotes that the effect of a factor was significant at $\alpha=0.05$, and the asterisks indicate the optimal level settings of significant design parameters for solely optimizing (maximizing) the $\mathrm{S} / \mathrm{N}$ ratio of an individual quality characteristic. Based on Table 12 , the 
optimal setting for a quality characteristic may conflict with the optimal setting for another quality characteristic. For example, a setting of $1.5^{\circ}$ for the design parameter $x_{3}$ is optimal for the maximum temperature $\left(y_{1}\right)$, but this is not the best choice for optimizing the material cost $\left(y_{3}\right)$. Based on a review of the factors' $\mathrm{S} / \mathrm{N}$ ratios and the suggestions of heat sink designers, the optimal design parameter settings were finally determined as shown in the last row in Table 12; Table 13 summarizes the simulation results for ten experimental trials. The first trial was carried out based on the optimal design parameter settings provided in Table 12; the remaining trials were implemented according to the nine possible combinations of the noise factors $z_{1}$ and $z_{2}$. According to Table 13, although the maximum temperature $\left(y_{1}\right)$ can fulfill its specifications, the thermal resistance $\left(y_{2}\right)$ in all experimental trials exceeded its upper specification limit. Furthermore, the average material cost $\left(y_{3}\right)$ was about 1.9 times the average material cost of a heat sink that was designed based on the optimal parameter settings obtained by the proposed cost-based optimization procedure. This proves that manually making tradeoffs to simultaneously optimize all quality characteristics is an inappropriate method for resolving a multiple-response parameter design problem.

Table 12. Analytical results from the Taguchi method.

\begin{tabular}{|c|c|c|c|c|c|c|c|c|c|c|c|c|c|c|c|c|c|}
\hline $\begin{array}{c}\text { Design } \\
\text { parameter }\end{array}$ & & $x_{1}$ & \multicolumn{3}{|c|}{$x_{2}(\mathrm{~W} / \mathrm{m} \cdot \mathrm{K})$} & \multicolumn{3}{|c|}{$x_{3}$ (degree) } & \multicolumn{3}{|c|}{$x_{4}(\mathrm{~mm})$} & \multicolumn{3}{|c|}{$x_{5}(\mathrm{~mm})$} & \multicolumn{3}{|c|}{$x_{6}$} \\
\hline $\mathrm{S} / \mathrm{N}$ for $y_{1}$ & $35.14 *$ & 34.89 & $35.17 *$ & 35.04 & 34.85 & $35.18 *$ & 34.98 & 34.89 & $35.57 *$ & 34.94 & 34.54 & $35.31 *$ & 34.99 & 34.76 & $35.44 *$ & 34.99 & 34.63 \\
\hline $\mathrm{S} / \mathrm{N}$ for $y_{2}$ & $10.66 *$ & 10.60 & $11.10 *$ & 10.83 & 9.97 & 10.64 & 10.61 & 10.64 & 10.61 & 10.66 & 10.63 & 10.63 & 10.63 & 10.63 & 10.61 & 10.64 & 10.64 \\
\hline $\mathrm{S} / \mathrm{N}$ for $y_{3}$ & 73.67 & 73.40 & 73.65 & 73.52 & 73.43 & 70.62 & 73.80 & $76.18 *$ & 71.48 & 73.54 & $75.57 *$ & 72.21 & 73.50 & $74.89^{*}$ & 72.20 & 73.54 & $74.85^{*}$ \\
\hline $\begin{array}{c}\text { Level } \\
\text { setting }\end{array}$ & 0.5 & 0.9 & 170 & 200 & 380 & 1.5 & 2.5 & 3.5 & 18 & 24 & 30 & 10.0 & 12.5 & 15.0 & 20 & 25 & 30 \\
\hline $\begin{array}{l}\text { Optimal } \\
\text { setting }\end{array}$ & \multicolumn{2}{|c|}{0.5} & \multicolumn{3}{|c|}{170} & \multicolumn{3}{|c|}{3.5} & \multicolumn{3}{|c|}{30} & \multicolumn{3}{|c|}{15.0} & \multicolumn{3}{|c|}{30} \\
\hline
\end{tabular}

\section{Conclusions}

To overcome shortcomings in the literature, including insufficient accuracy of second-order polynomials and subjective determination of relative weights and shape coefficients, this study applied gene expression programming (GEP), Taguchi quality loss, and particle swarm optimization (PSO) to develop a cost-based procedure for resolving a multi-response parameter design problem. The overall quality of a product was evaluated according to the actual material cost and visualized Taguchi quality loss due to deviations in quality characteristics from their targets. The feasibility and effectiveness of the proposed approach were verified through a case study on optimizing the design of a heat sink used for a high-power MR16 LED lamp. The experimental results indicated that the proposed solution procedure can provide highly robust design parameter settings for a heat sink that optimize the critical quality characteristics of the heat sink and minimize the actual material cost. Decision-makers no longer need to determine the relative weight of each response subjectively. Therefore, the approach proposed in this study can become a useful tool for resolving general multi-response parameter design problems in the real world. Analysis by the Taguchi method revealed that manually making tradeoffs to simultaneously optimize all quality characteristics is inappropriate for resolving a multiple-response parameter design problem.

The proposed procedure has several limitations. First, the GEP algorithm must be implemented for several runs due to its probabilistic evolutionary process; the final selected model for describing the mathematical relationship between quality characteristics and control factors cannot be proven as optimal. Second, the ideal values for each quality characteristic must be set subjectively through consultation with design engineers. Third, the parameter settings in PSO may influence the search efficiency and results; the optimal settings of these parameters are determined using an experimental design technique and are not guaranteed to be the most appropriate. Fourth, the proposed approach requires more experiment data in order to construct more accurate GEP models; however, the collection cost of the experiment data is not considered in the objective function. Finally, the optimal settings of design parameters provided by the PSO algorithm cannot be proven to be a real optimal design for a heat sink; the feasibility and effectiveness of the design can only be verified empirically. 
optimization of drilling parameters for CFRP composites with multiple performance characteristics, Measurement 45(5) (2012) 1286-1296.

9. A. K. Pandey and A. K. Dubey, Taguchi based fuzzy logic optimization of multiple quality characteristics in laser cutting of Duralumin sheet, Opt. Lasers Eng. 50(3) (2012) 328-335.

10. K. J. Kim and D. K. J. Lin, Simultaneous optimization of mechanical properties of steel by maximizing exponential desirability functions, J. Roy. Stat. Soc. C-App. 49 (2000) 311-325.

11. J. Kovach and B. R. Cho, Development of a multidisciplinary-multiresponse robust design optimization model, Eng. Optimiz. 40(9) (2008) 805-819.

12. M. Ramezani, M. Bashiri and A. C. Atkinson, A goal programming-TOPSIS approach to multiple response optimization using the concepts of non-dominated solutions and prediction intervals, Expert Syst. App. 38(8) (2011) 9557-9563.

13. S. Bera and I. Mukherjee, An adaptive penalty functionbased maximin desirability index for close tolerance multiple-response optimization problems, Int. J. $A d v$. Manuf. Tech. 61(1-4) (2012) 379-390.

14. S. P. Devi, S. Manivannan S and K. S. Rao, Comparison of nongradient methods with hybrid Taguchi-based epsilon constraint method for multiobjective optimization of cylindrical fin heat sink, Int. J. Adv. Manuf. Tech. 63(9-12) (2012) 1081-1094.

15. P. L. Goethals and B. R. Cho, Extending the desirability function to account for variability measures in univariate and multivariate response experiments, Comput. Ind. Eng. 62(2) (2012) 457-468.

16. Z. He, P. F. Zhu and S. H. Park, A robust desirability function method for multi-response surface optimization considering model uncertainty, Eur. J. Oper. Res. 221(1) (2012) 241-247.

17. R. Mukherjee, S. Chakraborty and S. Samanta, Selection of wire electrical discharge machining process parameters using non-traditional optimization algorithms, Appl. Soft. Comput. 12(8) (2012) 2506-2516.

18. A. Salmasnia, P. B. Kazemzadeh and M. M. Tabrizi, A novel approach for optimization of correlated multiple responses based on desirability function and fuzzy logics, Neurocomputing 91 (2012) 56-66.

19. C. Ferreira, Gene Expression Programming: Mathematical Modeling by an Artificial Intelligence (Springer, Berlin, Heidelberg, 2006).

20. Q. H. Feng, J. Y. Zhang, X. M. Zhang and A. M. Hu, Optimizing well placement in a coalbed methane reservoir using the particle swarm optimization algorithm, Int. J. Coal. Geol. 104 (2012) 34-45.

21. S. Kachroudi, M. Grossard and N. Abroug, Predictive driving guidance of full electric vehicles using particle swarm optimization, IEEE T. Veh. Technol. 61(9) (2012) 3909-3919.
22. S. Liu, R. Tao and C. M. Tam, Optimizing cost and CO2 emission for construction projects using particle swarm optimization, Habitat Int. 37 (2013) 155-162.

23. C. Ferreira, Gene expression programming: a new adaptive algorithm for solving problems, Compl. Syst. 13(2) (2001) 87-129.

24. M. Mitchell, An Introduction to Genetic Algorithms (MIT Press, Cambridge, Mass, 1996).

25. H. M. Azamathulla, A. A. Ghani, C. S. Leow, C. K. Chang and N. A. Zakaria, Gene-expression programming for the development of a stage-discharge curve of the Pahang River, Water Resour. Manag. 25(11) (2011) 2901-2916.

26. H. F. Li, M. Y. Lu, M. Zeng and B. Q. Huang, A nonparametric software reliability modeling approach by using gene expression programming, J. Inf. Sci. Eng. 28(6) (2012) 1145-1160.

27. L. Nie, X. Y. Shao, L. Gao and W. D. Li, Evolving scheduling rules with gene expression programming for dynamic single-machine scheduling problems, Int. J. $A d v$. Manuf. Tech. 50(5-8) (2010) 729-747.

28. K. K. Xu, Y. T. Liu, R. Tang, J. Zuo, J. Zhu and C. J. Tang, A novel method for real parameter optimization based on Gene Expression Programming, Appl. Soft Comput. 9(2) (2009) 725-737.

29. J. Kennedy and R. Eberhart, Particle swarm optimization, in Proc. 1995 IEEE Int. Conf. Neural Netw. (Perth, Western Australia, 1995), pp.1942-1948.

30. Y. H. Shi and R. Eberhart, A modified particle swarm optimizer, in Proc. 1998 IEEE Int. Conf. Evolu. Comput. (Anchorage, Alaska, 1998), pp. 69-73.

31. A. Abraham, H. Guo and H. Liu, Swarm intelligence: foundations, perspectives and applications, in Swarm Intelligent Systems, eds. N. Nedjah and L. M. Mourelle (Springer-Verlag, Berlin, 2006), pp. 3-25.

32. R. C. Eberhart and Y. Shi, Particle swarm optimization: developments, applications and resources, in Proc. 2001 IEEE C. Evolu. Computat. (Seoul, Korea, 2001), pp. 8186.

33. M. Clerc and J. Kennedy, The particle swarm - Explosion, stability, and convergence in a multidimensional complex space, IEEE T. Evolut. Comput. 6(1) (2002) 58-73.

34. M. V. Oliveira and R. Schirru, Applying particle swarm optimization algorithm for tuning a neuro-fuzzy inference system for sensor monitoring, Prog. Nucl. Energ. 51(1) (2009) 177-183.

35. N. Norouzi, R. Tavakkoli-Moghaddam, M. Ghazanfari, M. Alinaghian and A. Salamatbakhsh, A new multiobjective competitive open vehicle routing problem solved by particle swarm optimization, Netw. Spat. Econ. 12(4) (2012) 609-633.

36. S. P. Singh and A. R. Rao, Optimal allocation of capacitors in distribution systems using particle swarm optimization, Int. J. Eelc. Power. 43(1) (2012) $1267-$ 1275. 
37. J. Kennedy, R. C. Eberhart and Y. Shi, Swarm Intelligence (Morgan Kaufmann Publishers, San Francisco, 2001).

38. N. Nedjah and L. M. Mourelle, Swarm Intelligent Systems (Springer-Verlag, Berlin, 2006). 